\title{
Unmasking COVID-19 Vaccine "Infodemic" in the Social Media
}

\author{
John Demuyakor ${ }^{{ }^{*}}$ \\ (D) 0000-0002-6084-6951 SC 57223195290 AAF-2243-2021
}

\section{Isaac Newton Nyatuame ${ }^{2}$}

(iD) 0000-0001-7181-9521

\section{Samuel Obiri ${ }^{3}$}

(iD) 0000-0002-4314-5395

${ }^{1}$ Institute of Communication Studies, Communication University of China, Beijing, CHINA

2 Department of Communication Studies, University of Cape Coast, GHANA

${ }^{3}$ Department of Foreign Languages and Communication, University of Education, Winneba, GHANA

*Corresponding author: tevezkanzo@outlook.com

Citation: Demuyakor, J., Nyatuame, I. N., \& Obiri, S. (2021). Unmasking COVID-19 Vaccine "Infodemic" in the Social Media. Online Journal of Communication and Media Technologies, 11(4), e202119. https://doi.org/10.30935/ojcmt/11200

\section{ARTICLE INFO}

Received: 12 Jun 2021

Accepted: 29 Jul 2021

\section{ABSTRACT}

The activities of the anti-vaccine crusaders and conspiracy theorists on social media platforms have influenced billions of people across the globe on the safety of the COVID-19 vaccines currently been used for immunizations. The increased social media mis/disinformation on the efficacies and safety of these vaccines developed have raised a global concern among all stakeholders. This study adopted a convenience sampling through an online survey to collect data from $N=1800$ participants (students) from five (5) public universities in Accra the national capital of Ghana, which is also the epicenter of the COVID-19 pandemic to examine their perceptions on the overabundance COVID-19 pandemic information on social media and how these pieces information consumption impacts their trust/mistrust/distrust in the COVID-19 vaccines. The findings of our study indicate that there exist vital levels of COVID-19 vaccines "infodemic" on social media and these overabundances of COVID-19 vaccine information on social media has caused great levels of fear and panic among the students and the public. We also found that, the high exposure to COVID-19 vaccine information on social media are associated with negative perceptions and higher levels of mistrust/distrust among the population. This study recommends that stakeholders such as WHO, policymakers, and accredited health institutions must embark on public health communication campaigns to educate the public on the credible sources of COVID-19 pandemic information on social media.

Keywords: anti-vaccine crusaders, conspiracy theorists, COVID-19 vaccines, COVID-19 pandemic, misinformation, disinformation, social media, "infodemic"

\section{INTRODUCTION}

The World Health Organization (WHO) confirmed the COVID-19 pandemic as a global public health emergency due to the fast global spread in January 2020. WHO gave warning regarding "infodemic", which is a wave of fake news, misinformation, and disinformation on the deadly COVID-19 pandemic on various social media platforms (Germani \& Biller-Andorno, 2021; WHO, 2021 b). Since mid-2020 the hopes of getting COVID19 vaccines by the end of 2020 were very high. However, since the successful manufacturing, trial, and approval of these vaccines by the World Health Organization, experts are warning the global community about the increased wave of false news, misinformation, and disinformation on social media platforms that may affect acceptance and immunizations of the COVID-19 vaccines in order to keep people safe, informed, productive and connected (Gerts et al., 2021; Kang-Xing, 2020). While the majority of the global population relies on social media to keep connected and informed about the COVID-19 pandemic, and vaccines, the high

Copyright (c) 2021 by authors; licensee OJCMT. This article is an open access article distributed under the terms and conditions of the Creative Commons Attribution License (http://creativecommons.org/licenses/by/4.0/). 
volumes of "infodemic" on social media particularly Facebook, Twitter, and WhatsApp continue to undermine the global response and threaten measures to control the pandemic (Sanders, 2020; Tsao et al., 2021; Tseng, 2020).

In developing countries, like Ghana the net effects of fake news and mis/disinformation on COVID-19 vaccines are alarming (WHO, 2021a). Ghana became the first country outside of Africa to receive 600000 doses of COVID-19 vaccines in February 2021. Since the arrival of Ghana's first batch of COVID-19 vaccines via the Government of Ghana and the COVID-19 Vaccines Global Access, (COVAX Facility) initiated by the WHO, the conspiracy theories on the vaccines have increased on notable platforms like Facebook, Twitter, and WhatsApp. Therefore, the objective of this study is to examine students' perceptions of the overabundance of COVID-19 vaccines information on social media and how these volumes of information consumption impact students' trust/mistrust/distrust in the COVID-19 vaccines procured for nationwide immunizations.

\section{REVIEW OF RELATED LITERATURE}

\section{COVID-19 Vaccine "Infodemic" in the Social Media}

According to WHO, 2021 statistics, as of May 3, 2021, more than 3.19 million people have succumbed to the COVID-19 after it emerged towards the end of 2019. As of May 2021, the WHO had approve five vaccines for use across the globe. Besides these global consented efforts by the WHO, governments, and vaccine developers, the challenges of disbelief among the people on the development of vaccines within these short time and its accompany falsehood on the negative impacts of the vaccines.

A joint statement by WHO et al. (2020), regarding the management of COVID-19 pandemic, defined "infodemic", as the overabundance of information, which entailed deliberate attempt to distribute wrong information. Since the vaccinations began, different groups have emerged on social media and made the spreading of misinformation about COVID-19 vaccines and mass immunizations across the globe (Bogart et al., 2021; Paul et al., 2021; Prieto Curiel \& González Ramírez, 2021). In January 2020 the WHO has reported that fake news, disinformation, and misinformation threatens COVID-19 vaccines acceptance and COVID-19 vaccination plans across the world. Again, WHO et al. (2020), also believe that, without the right information, the campaigns about immunization may not be able to meet the targeted level and the virus can continue to thrive (WHO, 2021a). With approval from the WHO, some vaccine developers such as Sinovac, Sinopharm, Pfizer/BioNTech, Moderna, and AstraZeneca/Oxford University are playing the leading role in keeping the population informed on the benefits of the vaccines produced. Already, several governments globally have procured the COVID-19 vaccines, and are carrying out vaccinations among the general civilian populations and especially for the vulnerable groups (WHO, 2021a).

According to a recent study by Tsao et al. (2021), Mega (2021), Jennings et al. (2021), Hall Jamieson and Albarracín (2020), the popular social media platforms acting as vectors and are noted for promoting wrong information and unfounded news regarding the COVID-19 vaccines are Facebook, Twitter, YouTube, and WhatsApp. Equally, most anti-disinformation websites, suggested that social media platforms have not just highlighted the level of mis/disinformation across the world but also pointed out the negative impacts that mis/disinformation can have on vaccines, institutions, and scientific findings (Gerts et al., 2021; Loomba et al., 2021).

The immunization department of the World Health Organization reported that, the agency is worried about the mis/disinformation that was being propagated by the anti-vaccines movements and that there was a possibility of dissuading people from being immunized against the virus. The WHO's immunization department further indicated that, it was very concerned about how and where people received critical information on vaccines. The department cautioned people to be aware that there is a lot of wrong information out there, either intentionally or unintentionally (WHO, 2021b).

A recent study published in British Medical Journal and authored by Wilson and Wiysonge (2020), on "Social Media and Vaccine Hesitancy" pointed out that there exist links between disinformation, online campaigns, COVID-19 vaccines acceptances and vaccinations. According to Wilson and Wiysonge (2020), Chadwick et al. (2021), Latkin et al. (2021), and Loomba et al. (2021), the impact created by mis/disinformation on social media 
regarding COVID-19, vaccines, and vaccinations have increased the number of people who are hesitant to get the vaccine, even though their fear has no scientific backing.

\section{COVID-19 Vaccine Conspiracy Theories in the Social Media}

Many countries have lunch aggressive implementation plans to procure COVID-19 vaccines, and vaccinate their population. The WHO reported that as of $3^{\text {rd }}$ May 2021 a total of 1.15 billion have been given doses and 273 million fully vaccinated and translates to 3.5\% of the global population (WHO, 2021a).

The COVID-19 vaccines approved by WHO for emergency use, and vaccination programs are labeled with so many conspiracy theorists. One group of conspiracy theorists say the COVID-19 vaccines have implanted microscopic chips for monitoring recipients of the vaccine(s) (Allington et al., 2020; Gerts et al., 2021). Such kinds of reasoning can thrive especially when the polls show that the high levels of superstition and illiteracy in developing countries like Ghana, where the people are already doubtful about getting immunized. Other concerns are that, the vaccines have been developed within a short time, without studies about the efficacy and possible side-effects.

A study carried out by Ipsos in November 2020, on how "infodemic" risks jeopardizing COVID-19 vaccines contended that even in developed nations like France, with a high literacy rate, $54 \%$ of the French people were unwilling to be immunized against COVID-19. The figure is 10\% lower than the US, 22 percent lower than Canada, and 33\% lower than that of India (Lazarus et al., 2021).

Also, in a study conducted in 15 countries, an average of $73 \%$ of the people agreed that they were unwilling to be vaccinated against the virus, a figure that is $4 \%$ lower than the initial study carried out by (Boyon, 2020). Besides the vaccine's conspiracy theorists, more and more people are continuing to express mistrust over the institutions that are mandated to implement the immunization process (Allington et al., 2020; Gerts et al., 2021). The theories around the conspiracy are that "our elites are lying to us." All institutions of authority, both from the government and scientific community have experienced a growing mistrust due to misinformation. When people are not able to receive trusted information regarding the COVID-19 vaccines and mistrust engulfs, the main actors and institutions that are supposed to play a leading role in the immunization process, very little can be achieved (Cinelli et al., 2020; Kang-Xing, 2020; Smith et al., 2020; Wardle \& Singerman, 2021). Based on the reviewed literature, and gaps identified, the following Research Questions (RQs), will guide this study.

\section{Research Questions (RQs)}

RQ1. What are the reactions of university students to COVID-19 vaccine information on social media?

RQ2. What is the level of trust/mistrust among university students on COVID-19 vaccine information they read on social media?

RQ3. What are some of the sources of credible sources of COVID-19 vaccine information on social media?

\section{MATERIALS AND METHODS}

This study adopted an online survey through convenience sampling to assess university students' perceptions of the overabundance of COVID-19 vaccines information on social media and how these volumes of information consumption impact their trust/mistrust in the COVID-19 vaccines. The researchers resorted to an online survey due to the COVID-19 restrictions imposed by the government of Ghana for pandemic control. The study was conducted in five conveniently sampled universities in Accra, which is the hub of most universities in Ghana and also the epicenter of the COVID-19 pandemic in Ghana. The universities included in the survey were, the University of Ghana, Ghana Institute of Management and Public Administration, University of Professional Studies, Ghana Institute of Journalism, and African University College of Communications. Students who were over 18 years and have continuously lived in Accra since the first reported case of COVID-19 on March 12, 2020 and were, eligible to participate in the study. One of the reason behind the reference period is to target students who have a fair knowledge of how the COVID-19 started in Ghana and vaccine information. 
The study was carried out online from 20 February 2021 to May 2021. Before the instruments (questionnaire) were administered to the target population, the researchers first pilot-tested the questionnaire on the WhatsApp group pages of 50 students (the respondents) who had a similar socioeconomic background in the Kwame Nkrumah University in Kumasi, the capital of the Ashanti region of Ghana (which was not part of the sample) to ensure its validity and reliability and ensure research that the ethics are well adhered to. This pilot group were asked to complete the online questionnaire and provide comments regarding their understanding of the questions. The feedback from the questionnaire resulted in some modifications. The questionnaire were structured into three sections, through the use of a five-point Likert. The three sections of the questionnaire are; socio-demographics, the reactions of students to COVID-19 vaccines information on social media, and the level of trust/mistrust about COVID-19 vaccines information they read on social media. The socio-demographic variables include age, sex, education, employment status, and type of social media platform(s) used. The link for the questionnaire were sent via WhatsApp to various students' group platforms in the sampled universities. After informed concerns from WhatsApp group administrators in the sample schools, participants subsequently agreed before partaking in the survey. The online survey recorded 1800 participants. The final data was corded and percentages, standard deviations, and mean were used for the final analysis.

\section{Measurement}

The measure identified three variables as the primary quantitative outcomes; Reactions to social media information about coronavirus vaccine, level of trust of COVID-19 vaccine information on social media, and sources of credible COVID-19 Vaccine information on social media.

\section{Reactions to COVID-19 vaccine information on social media}

The first primary quantitative outcome was reactions to the COVID-19 vaccine information on social media, which were assessed with the following variables; "Happy, "Amused, "Excited, "Angry, "Sad, and "Panicking. The common response options for each of the variables were; "Strongly Agree," "Agree," "Neither agree nor disagree," "Disagree," and "Strongly disagree."

\section{Level of trust/distrust of COVID-19 vaccines information on social media}

The second quantitative primary variable was the level of trust/distrust of COVID-19 vaccines information on social media platforms, which was evaluated with the following questions; "I do not trust COVID-19 vaccines information on social media" and, "I greatly trust COVID-19 information on social media". As a way of examining the reasons for vaccine mistrust, open-ended secondary questions were asked. The questions included; "Why would you not trust COVID-19 vaccine information on social media?" The question was only directed to the respondents who answered, "Neither agree nor disagree," "Agree," and "Strongly agree" to the primary question, "I do not trust COVID-19 vaccine information on social media" $(\mathbf{N}=\mathbf{1 8 0 0})$.

\section{Sources of credible COVID-19 Vaccine information on social media}

The third quantitative variable was sources of reliable COVID-19 vaccine information on the social media platform(s), "WHO social media handles and websites", "CDC/ACDC social media handles and websites", "National Institutes of Health/Ghana health service social media handles and websites", "Credible academic institutions, and large healthcare organizations", "links and references to peer-reviewed research", and "Established medical journals".

\section{ANALYSIS AND RESULTS}

\section{Demographic Characteristics of Respondents}

The three demographic characteristics analyzed were gender, age, and level of academic qualification. The type of social media platform(s) used by students were also analyzed. Table 1 shows the detailed demographic information on students from the five sampled universities for this study. The ages ranged from 18-36; there were 989 (54.9\%) females and 811 (45.1\%) males. In all, 235 (13.1\%) are in level 100; with 432 (24\%) level 200 students; Level 300 students were 567 (31.5\%) and 566 (31.4\%) were level 400 students. Even 
Table 1. Descriptive statistics of respondents

\begin{tabular}{|c|c|c|c|}
\hline Demographic Variables & Subgroups & Frequency $(\mathrm{N}=317)$ & Percentage (100\%) \\
\hline \multirow[t]{4}{*}{ Age } & $<18$ & 467 & 25.9 \\
\hline & $18-24$ & 678 & 37.7 \\
\hline & $25-35$ & 325 & 18.1 \\
\hline & $>35$ & 330 & 18.3 \\
\hline \multirow[t]{2}{*}{ Gender } & Male & 989 & 54.9 \\
\hline & Female & 811 & 45.1 \\
\hline \multirow[t]{4}{*}{ Education } & Level 100 & 235 & 13.1 \\
\hline & Level 200 & 432 & 24 \\
\hline & Level 300 & 567 & 31.5 \\
\hline & Level 400 & 566 & 31.4 \\
\hline \multirow[t]{5}{*}{ Social media type use } & Facebook & 943 & 52.3 \\
\hline & Twitter & 98 & 5.5 \\
\hline & WhatsApp & 641 & 35.6 \\
\hline & YouTube & 100 & 5.6 \\
\hline & Others & 18 & 1 \\
\hline
\end{tabular}

Table 2. Reactions to COVID-19 vaccine information on social media

\begin{tabular}{llcr}
\hline Item & Specific Reactions & Mean & Std. Deviation \\
\hline 1 & Happiness & 2.34 & 1.50 \\
2 & Amused & 3.64 & 1.59 \\
3. & Excited & 3.37 & 1.55 \\
4. & Angry & 3.38 & 1.44 \\
5. & Sadness & 3.62 & 1.52 \\
6. & High levels of Panic and Fear & 4.11 & 2.35 \\
\hline
\end{tabular}

Table 3. Descriptive statistics of the level of trust of COVID-19 vaccines information on social media

\begin{tabular}{llcc}
\hline Item & Trust/distrust Levels of participants & Mean & Std. Deviation \\
\hline 1 & I do not trust COVID-19 vaccines information on social media & 4.00 & 1.31 \\
2 & I greatly trust COVID-19 information on social media & 3.96 & 1.29 \\
\hline
\end{tabular}

though the social media used by students were not considered as the objective of this, the researchers decided to add that section to get a snapshot of social media platforms mostly used by students for COVID19 vaccine "infodemic" on social media. The following were details of the analysis of the result; Facebook (52.3\%); Twitter (5.5\%); WhatsApp (35.6\%); YouTube (5.6\%), and Others (1\%). See Table 1.

\section{The Reactions to COVID-19 Vaccine Information on Social Media}

To determine the student's reactions to information on social media regarding the COVID-19 vaccines, a five-scale -Likert types of questions were asked (Table 2). The highest response of students reactions towards COVID-19 vaccines information on social media was high levels of panicking and fear $(4.11 \pm 2.35)$ and sadness $(3.62 \pm 1.52)$ as the second highest reactions of respondents. See Table 2 for details.

\section{The Level of Trust/Distrust of COVID-19 Vaccines Information on Social Media}

One of the prime research questions was to investigate the levels of trust/distrust of COVID-19 vaccine information on social media. Therefore, we solicited views from the respondents on the possible level of trust/distrust of COVID-19 vaccines. Our study found out that majority of respondents cited that "they

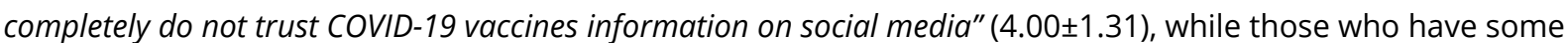
trust in the information about COVID-19 vaccines are (3.96 \pm 1.29$)$. See Table 3 for details.

\section{The Reasons for Mistrust/ Distrust of COVID-19 Vaccines Information on Social Media}

One of the goals of this study was to identify the reasons for mistrust of COVID-19 vaccine information on social media. Therefore, we solicited views from the respondents on possible reasons for mistrust/ distrust of COVID-19 vaccines information on social media. The most prominent reasons for COVID-19 vaccine mistrust are "Too much Information on COVID-19 Vaccine Hesitancy on social media" (4.11 \pm 2.35$)$, and "Too much 
Table 4. Descriptive statistics on the reasons for mistrust/distrust of COVID-19 vaccines information on social media

\begin{tabular}{llcc}
\hline Item & Reasons for mistrust/ distrust of COVID-19 vaccines & Mean & Std. Deviation \\
\hline 1 & Too much different social media information on vaccine makers & 3.89 & 1.42 \\
2 & Too much exposure to different social media for COVID-19 news & 2.76 & 1.52 \\
3. $\quad \begin{array}{l}\text { Too much social media information the risk perceptions and safety of } \\
\text { the COVID-19 vaccines }\end{array}$ & 4.00 & 1.31 \\
4. $\quad$ Too much Information on COVID-19 Vaccine Hesitancy on social media & 4.11 & 2.35 \\
\hline
\end{tabular}

Table 5. Descriptive statistics on the sources of credible COVID-19 vaccine information

\begin{tabular}{llcc}
\hline Item & Sources of credible COVID-19 Vaccine & Mean & Std. Deviation \\
\hline 1 & WHO social media handles and websites & 3.91 & 1.33 \\
2 & CDC/ACDC social media handles and websites & 3.76 & 1.53 \\
3. & National Institutes of Health/Ghana health service social media handles & 3.37 & 1.75 \\
& and websites & 3.04 & 1.11 \\
4. & Credible academic institutions and large healthcare organizations & 2.86 & 1.46 \\
5. & Vaccines information on mainstream public social media platforms & 2.96 & 1.21 \\
6. & Established medical journals and peer-reviewed research & \\
\hline
\end{tabular}

exposure to different social media platforms for COVID-19 news" (2.76 \pm 1.52$)$, is the least reason for COVID-19 vaccines mistrust. See Table 4 for details.

\section{Sources of Credible Coronavirus Vaccine Information on Social Media}

On the sources of credible COVID-19 vaccines information on various media platforms, our study reported that the majority of respondents opted for COVID-19 vaccines information from "WHO social media handles and websites" (3.91 \pm 1.33 ), while the least source of credible COVID-19 vaccine information is from "links and

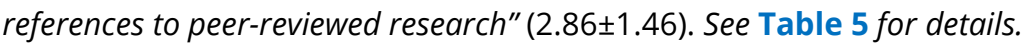

\section{DISCUSSION}

While the global community struggles to get trusted news on the vaccine, several others used social media platforms to send fear and panic to the people who are supposed to receive reliable and vital news on the vaccines during these difficult times. Our findings show that social media generates the highest sources of COVID-19 "infodemic" and related Conspiracy theories. In terms of social media platforms, and conspiracy theory-related content, our study reported that Facebook content accounted for $52.3 \%$ of the COVID-19 vaccine "infodemic", compared with other platforms like WhatsApp, Twitter, and YouTube.

On the reactions of students to COVID-19 vaccine information on social media, the researchers found out that, respondents' reactions to any information on social media about the COVID-19 vaccines is that of high fear and panic (4.11 \pm 2.35$)$. The reports of constantly edited screenshots of blood splattered around in decrepit hospital wards when "the first COVID-19 vaccine patients begin to consume other patients,". These confirm the findings of Wilson and Wiysonge (2020), and Loomba et al. (2021) on people's reactions of fear and panic to COVID-19 vaccines on social media.

We found that high exposure to COVID-19 vaccine information on social media platforms is linked to higher risk of negative perceptions. In addition, the trust levels regarding the vaccines on social media platforms have become contentious among the experts as well as the public. Though scientists are not so sure about the possibilities of long-term effects that are likely to be caused by the COVID-19 vaccines, there have been some instances where people have experienced adverse effects after getting vaccinated. COVID19 vaccines complications have further heightened the level of fear and distrust levels of respondents. Our findings of trust/distrust levels of the COVID-19 vaccines on social media show that a great number of our

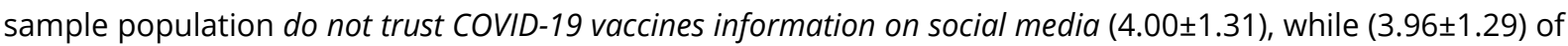
the respondents indicated they have some amount of trust for COVID-19 vaccines information on social media. These findings confirm studies by Mega (2021), Loomba et al. (2021), and Jennings et al. (2021) who reported a high level of mistrust/distrust for the COVID-19 vaccine information on social media. 
According to our study, some of the reasons assigned for the great mistrust/distrust of these COVID-19 vaccines information on social media include; too much different social media information on vaccine makers, too much exposure to different social media for COVID-19 news, too much social media information the risk perceptions and safety of the COVID-19 vaccines, too much Information on COVID-19, and too much information on Vaccine hesitancy. These findings are in line with previous studies conducted on vaccine hesitancy by Sonawane et al. (2021), Ipsos (2020b), Su et al. (2020). Also, our study releveled that the fake news on social media, on the reaction from people purported to have inoculated the COVID-19 vaccines have also greatly contributed to the high levels of mistrust for COVID-19 vaccines, therefore, reducing COVID-19 vaccines mistrust/distrust on social media must be a global priority.

Our last research question was to investigate the sources of credible COVID-19 vaccines information on social media. Before considering vaccine information on social media our study listed the following sources as the most credible outlets for COVID-19 vaccine; WHO social media handles and websites, CDC/ACDC social media handles and websites, national institutes of health/Ghana health service social media handles, credible academic institutions and large healthcare organizations, links and references to, and established medical journals and peer-reviewed research, and public social media platforms. Our respondents cited WHO social media handles and websites as their first-choice sources of credible and reliable information on COVID-19 vaccines and public and private social media platforms as the least credible source of COVID-19 vaccines information. These findings are backed by studies conducted by WHO (2021), Cinelli et al. (2020), Larson (2018), Viswanath et al. (2021) on credible sources of other viral diseases, COVID-19 social media "infodemic", and social determinants of the COVID-19 vaccine respectively.

\section{CONCLUSION}

In sum, the findings from our study show that there are high levels of the COVID-19 vaccine "infodemic" on social media. This overabundance of COVID-19 information on social media has caused great levels of panic, fear, and mistrust/distrust among the general public. To reduce this mis/disinformation regarding COVID-19 vaccines especially on social media stakeholders, such as WHO, policymakers, and accredited health institutions need to find an innovative ways to address the overabundance of COVID-19 vaccine information on social media. Governments and stakeholders can undertake critical initiatives such as implanting critical policy decisions on misinformation and disinformation, and carrying out massive public education on factchecking in order to reduce the level of panic, fear, and mistrust/distrust that the overabundance of COVID19 information on social media has brought to the public.

The findings of our study has pedagogical importance for the teaching of COVID-19 "infodemic" on social media. This study will equip scholars, and early researchers within the academia to have a first-hand knowledge, on how to become aware of the strategies of identifying misinformation and disinformation on social media. Again, this study contributes to the academic literature on COVID-19 vaccine "infodemic" on social media in developing countries like Ghana (West Africa) and other emerging economics by demonstrating the usefulness and limitations of social media "infodemic" on the success or failure of vaccines immunizations programs been carried out across the globe. This study also add-up to the existing literature on citizen's perceptions of the COVID-19 vaccine "infodemic" on social media, in terms of the benefits and challenges citizens go through in accessing the COVID-19 vaccines information. Therefore, this article documents how the COVID-19 vaccines information on social media can be used to promote the socioeconomic development of Ghana and other developing nations across the world. Our findings will also aid various governments across the globe to put in pragmatic measures to manage the overabundance of COVID19 pieces of information in social media.

Author contributions: All authors were involved in concept, design, collection of data, interpretation, writing, and critically revising the article. All authors approve final version of the article.

Funding: The authors received no financial support for the research and/or authorship of this article.

Declaration of interest: Authors declare no competing interest.

Data availability: Data generated or analysed during this study are available from the authors on request. 


\section{REFERENCES}

Allington, D., Duffy, B., Wessely, S., Dhavan, N., \& Rubin, J. (2020). Health-protective behaviour, social media usage and conspiracy belief during the COVID-19 public health emergency. Psychological Medicine, 51(10), 1763-1769. https://doi.org/10.1017/S003329172000224X

Bogart, L. M., Ojikutu, B. O., Tyagi, K., Klein, D. J., Mutchler, M. G., Dong, L., Lawrence, S. J., Thomas, D. R., \& Kellman, S. (2021). Covid-19 related medical mistrust, health impacts, and potential vaccine hesitancy among black Americans living with HIV. JAIDS Journal of Acquired Immune Deficiency Syndromes, 86(2), 200207. https://doi.org/10.1097/QAI.0000000000002570

Boyon, N. (2020). COVID-19 vaccination intent is decreasing globally. Ipsos. https://www.ipsos.com/en/globalattitudes-covid-19-vaccine-october-2020

Chadwick, A., Kaiser, J., Vaccari, C., Freeman, D., Lambe, S., Loe, B. S., Vanderslott, S., Lewandowsky, S., Conroy, M., Ross, A. R. N., Innocenti, S., Pollard, A. J., Waite, F., Larkin, M., Rosebrock, L., Jenner, L., McShane, H., Giubilini, A., Petit, A., \& Yu, L.-M. (2021). Online social endorsement and covid-19 vaccine hesitancy in the united kingdom. Social Media + Society, 7(2), 205630512110088. https://doi.org/10.1177/ 20563051211008817

Cinelli, M., Quattrociocchi, W., Galeazzi, A., Valensise, C. M., Brugnoli, E., Schmidt, A. L., Zola, P., Zollo, F., \& Scala, A. (2020). The COVID-19 social media infodemic. Scientific Reports, 10(1), 16598. https://doi.org/10.1038/s41598-020-73510-5

Germani, F., \& Biller-Andorno, N. (2021). The anti-vaccination infodemic on social media: A behavioral analysis. PLOS ONE, 16(3), e0247642. https://doi.org/10.1371/journal.pone.0247642

Gerts, D., Shelley, C. D., Parikh, N., Pitts, T., Ross, C. W., Fairchild, G., Chavez, N. Y. V., \& Daughton, A. R. (2021). "Thought i'd share first" and other conspiracy theory tweets from the covid-19 infodemic: Exploratory study. JMIR Public Health and Surveillance, 7(4), e26527. https://doi.org/10.2196/26527

Hall Jamieson, K., \& Albarracín, D. (2020). The relation between media consumption and misinformation at the outset of the sars-cov-2 pandemic in us. Harvard Kennedy School Misinformation Review. https://doi.org/10.37016/mr-2020-012

Ipsos. (26 November 2020a). "Infodemic" risks jeopardizing virus vaccines. https://malaysia.news.yahoo.com/infodemic-risks-jeopardising-virus-vaccines-082447684.html

Ipsos. (October, 2020b). COVID-19 vaccination intent is decreasing globally. https://www.ipsos.com/en/globalattitudes-covid-19-vaccine-october-2020

Jennings, W., Stoker, G., Willis, H., Valgardsson, V., Gaskell, J., Devine, D., McKay, L., \& Mills, M. C. (2021). Lack of trust and social media echo chambers predict COVID-19 vaccine hesitancy. Health Policy. https://doi.org/10.1101/2021.01.26.21250246

Kang-Xing, J. (2020, December 18). Keeping people safe and informed about the coronavirus. About Facebook. https://about.fb.com/news/2020/12/coronavirus/

Latkin, C. A., Dayton, L., Yi, G., Konstantopoulos, A., \& Boodram, B. (2021). Trust in a COVID-19 vaccine in the U.S.: A social-ecological perspective. Social Science \& Medicine, 270, 113684. https://doi.org/10.1016/j.socscimed.2021.113684

Larson, H. J. (2018). The biggest pandemic risk? Viral misinformation. Nature, 562(7727), $309-309$. https://doi.org/10.1038/d41586-018-07034-4

Lazarus, J. V., Ratzan, S. C., Palayew, A., Gostin, L. O., Larson, H. J., Rabin, K., Kimball, S., \& El-Mohandes, A. (2021). A global survey of potential acceptance of a COVID-19 vaccine. Nature Medicine, 27(2), 225-228. https://doi.org/10.1038/s41591-020-1124-9

Loomba, S., de Figueiredo, A., Piatek, S. J., de Graaf, K., \& Larson, H. J. (2021). Measuring the impact of COVID19 vaccine misinformation on vaccination intent in the UK and USA. Nature Human Behaviour, 5(3), 337348. https://doi.org/10.1038/s41562-021-01056-1

Mega, E. R. (2021). Trust in COVID vaccines is growing. Nature, d41586-021-00368-6. https://doi.org/10.1038/d41586-021-00368-6

Paul, E., Steptoe, A., \& Fancourt, D. (2021). Attitudes towards vaccines and intention to vaccinate against COVID-19: Implications for public health communications. The Lancet Regional Health - Europe, 1, 100012. https://doi.org/10.1016/j.lanepe.2020.100012 
Prieto Curiel, R., \& González Ramírez, H. (2021). Vaccination strategies against COVID-19 and the diffusion of anti-vaccination views. Scientific Reports, 11(1), 6626. https://doi.org/10.1038/s41598-021-85555-1

Sanders, A. C., White, R. C., Severson, L. S., Ma, R., McQueen, R., Alcântara Paulo, H. C., Zhang, Y., Erickson, J. S., \& Bennett, K. P. (2020). Unmasking the conversation on masks: Natural language processing for topical sentiment analysis of COVID-19 Twitter discourse. Health Informatics. https://doi.org/10.1101/2020.08.28.20183863

Smith, R., Cubbon, S. \& Wardle, C. (2020). Under the surface: Covid-19 vaccine narratives, misinformation \& data deficits on social media. First Draft. https://firstdraftnews.org/vaccinenarratives- full-report-November2020

Sonawane, K., Troisi, C. L., \& Deshmukh, A. A. (2021). COVID-19 vaccination in the UK: Addressing vaccine hesitancy. The Lancet Regional Health - Europe, 1, 100016. https://doi.org/10.1016/j.lanepe.2020.100016

Su, Z., Wen, J., Abbas, J., McDonnell, D., Cheshmehzangi, A., Li, X., Ahmad, J., Šegalo, S., Maestro, D., \& Cai, Y. (2020). A race for a better understanding of COVID-19 vaccine non-adopters. Brain, Behavior, \& Immunity - Health, 9, 100159. https://doi.org/10.1016/j.bbih.2020.100159

Tseng, B. (17th December 2020). Combatting vaccine misinformation in the age of the internet. Institute for Global Change. https://institute.global/policy/combatting-vaccine-misinformation-age-internet

Tsao, S.-F., Chen, H., Tisseverasinghe, T., Yang, Y., Li, L., \& Butt, Z. A. (2021). What social media told us in the time of COVID-19: A scoping review. The Lancet Digital Health, 3(3), e175-e194. https://doi.org/10.1016/S2589-7500(20)30315-0

Viswanath, K., Bekalu, M., Dhawan, D., Pinnamaneni, R., Lang, J., \& McLoud, R. (2021). Individual and social determinants of COVID-19 vaccine uptake. BMC Public Health, 21(1), 818. https://doi.org/10.1186/s12889021-10862-1

Wardle, C., \& Singerman, E. (2021). Too little, too late: Social media companies' failure to tackle vaccine misinformation poses a real threat. BMJ, n26. https://doi.org/10.1136/bmj.n26

WHO. (2021a, March 31). Viral Facts Africa initiative to combat dangerous health misinformation. Africa Renewal. https://www.un.org/africarenewal/news/viral-facts-africa-initiative-combat-dangerous-healthmisinformation

WHO. (2021b, 27 April). Fighting misinformation in the time of COVID-19, one clicks at a time. https://www.who.int/ news-room/feature-stories/detail/fighting-misinformation-in-the-time-of-covid-19-one-click-at-a-time

WHO, UN, UNICEF, UNDP, UNESCO, UNAIDS, ITU, UN Global Pulse, and IFRC, (2020). Managing the COVID-19 infodemic: Promoting healthy behaviours and mitigating the harm from misinformation and disinformation.https://www.who.int/news/item/23-09-2020-managing-the-covid-19-infodemicpromoting-healthy-behaviours-and-mitigating-the-harm-from-misinformation-and-disinformation

Wilson, S. L., \& Wiysonge, C. (2020). Social media and vaccine hesitancy. BMJ Global Health, 5(10), e004206. https://doi.org/10.1136/bmjgh-2020-004206

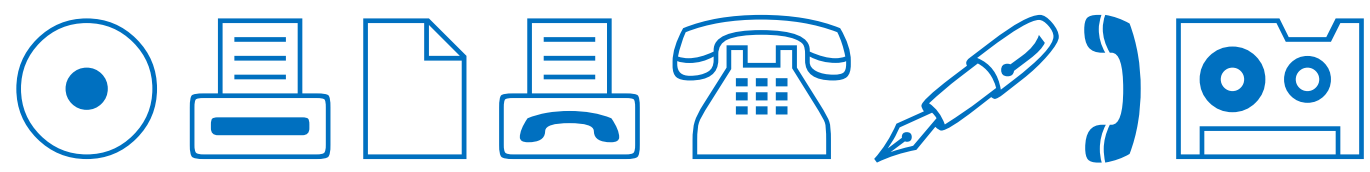

